\title{
In Memory of Tachycardia: A wide complex tachycardia in a young male
}

\author{
Gregory Siroky ${ }^{1}$, Seth Keller ${ }^{1}$, and Ranjit Suri ${ }^{1}$ \\ ${ }^{1}$ Mount Sinai Health System
}

May 11, 2020

\section{EP Rounds}

A 24-year-old male with no past medical history presents to an urgent care center with palpitations. Four days prior he noticed that his heart rate was elevated and sometimes irregular which he recorded on his fitness watch. He had associated intermittent diaphoresis and dyspnea with exertion. Initial ECG obtained is shown in Figure 1A. He was instructed to follow up with a cardiologist. Transthoracic echocardiogram (TTE) obtained in the office demonstrated global left ventricular (LV) dysfunction with an ejection fraction (EF) of $35 \%$. After being sent to the hospital, administration of intravenous amiodarone terminated the patient's tachycardia and a subsequent ECG was obtained (Figure 1B). Repeat TTE showed normalization of the $\mathrm{EF}$ to $55 \%$ and cardiac magnetic resonance imaging (cMRI) demonstrated a normal LV without evidence of myocardial pathology. What is the most likely diagnosis of the tachycardia? What is the etiology of the abnormalities seen in the patient's ECG as shown in Figure 1A? What is the appropriate management for this patient?

\section{Discussion}

The initial ECG shows a wide complex tachycardia (WCT) at $160 \mathrm{bpm}$, left axis deviation of -35, QRS duration of 146ms and morphology demonstrating a right bundle branch (RBBB) and left anterior fascicular block (LAFB) pattern. The differential diagnosis for such a tachycardia includes a supraventricular tachycardia (SVT) with RBBB/LAFB aberrancy, an SVT with bystander nodofascicular (NF) accessory pathway (AP) connecting the slow AV nodal pathway to the left posterior fascicle,${ }^{1}$ an orthodromic reentrant tachycardia (ORT) using a concealed $\mathrm{NF} \mathrm{AP}^{2}$, or a left posterior fascicular (LPF) ventricular tachycardia (VT) using the LPF as the retrograde limb of the circuit. Inspection of the ECG shows atrio-ventricular (AV) dissociation, which can be seen in ORT with a NF AP (usually with a 1:2 AV relationship; not seen in our patient's ECG), however, combined with the presence of fusion complexes, the diagnosis of LPF VT is more likely (Figure 2). Electrophysiologic study confirmed the diagnosis of LPF VT, however, the arrhythmia was difficult to induce and when induced it was non-sustained. Therefore, anatomic-based ablation ${ }^{3}$ of the distal one third of the LPF was performed (Supplementary Figure), rendering the tachycardia non-inducible. An additional endpoint was the appearance of new q-waves in the inferior limb leads (II, III, aVF) as a result of ablating the distal third of the LPF (Figure 3).

Now turning attention to the abnormalities seen in the baseline ECG, specifically the inferior/anterolateral T-wave inversions (TWI). There are numerous causes of TWI, most which are of clinical concern and an emergency, however, there are a few etiologies which are less sinister and quite benign. A clinician seeing these abnormalities in conjunction with a diagnosis of VT, would be concerned with myocardial ischemia thereby triggering a cascade of events leading to coronary artery evaluation and exposing the patient to unnecessary testing and potential risks. Taking into consideration the patient's age, palpitations, and lack of chest pain, myocardial ischemia is less likely. In light of the diagnosis of VT, the more reasonable cause of the TWI is cardiac memory, or T-wave memory (TWM). Cardiac memory results from abnormal ventricular 
activation leading to short- and long-term alterations on the molecular level by which repolarization changes are achieved and can occur after ventricular pacing, after resolution of left bundle branch block, after ablation of an AP, and post termination of VT. ${ }^{4,5}$ It can sometimes be challenging to differentiate TWI as a result of TWM versus myocardial ischemia, however, Shvilkin et al. proposed two criteria in favor of TWM: 1) positive T-waves in leads I and aVL, and 2) precordial TWI > inferior TWI, both of which fit the patient's ECG. ${ }^{5}$ ECG obtained during follow-up visit demonstrated resolution of the TWI further confirming the diagnosis of TWM.

In conclusion, this case highlights three important concepts: 1) the correct diagnosis of a WCT is imperative to a patient's management, 2) TWI are not always pathologic but can be physiologic, and 3) T-waves always "remember" abnormal ventricular activation and can be a useful diagnostic tool in assessing new TWI.

\section{Sources of funding: None}

\section{Disclosures: None}

\section{References}

1. Hoffmayer K, Lee B, Vedantham V, et al. Variable Clinical Features and Ablation of Manifest Nodofascicular/Ventricular Pathways. Circ Arrhythm Electrophysiol. 2015; 8: 117-127.

2. Sternick E and Wellens HJ. Variants of Ventricular Preexcitation. Massachusetts: Blackwell Publishing; 2006: p. 79 .

3. Lin D, Hsia H, Gerstenfeld E, et al. Idiopathic fascicular left ventricular tachycardia: Linear ablation lesion strategy for noninducible or nonsustained tachycardia. Heart Rhythm. 2005; 2: 934-939.

4. Wellens HJ. QRS Complex change during carotid sinus massage in ventricular pre-excitation. Heart Rhythm. Aug 2019; 16 (8): 1296-1298.

5. Shvilkin A, Huang H, Josephson ME. Cardiac memory: Diagnostic Tool in the Making. Circ Arrhythm Electrophysiol. 2015; 8(2): 475-482.

\section{Figure Legends}

Figure 1. (A) Initial 12-lead ECG obtained at urgent care center. (B) Repeat 12-lead ECG after cessation of tachycardia.

Figure 2. Initial 12-lead ECG demonstrating AV dissociation (arrows) and fusion complexes (stars) making ventricular tachycardia (VT) the clear diagnosis.

Figure 3. Pre- (A) and post-ablation (B) ECGs demonstrating new q-waves in the inferior limb leads (II, III, and aVF) representing ablation of the distal portion of the left posterior fascicle with initial forces directed superiorly.

Supplementary Figure. Electroanatomic map of left ventricular septum as viewed from the right anterior oblique projection (RAO). Areas of the His cloud (yellow dots), left anterior fascicle (LAF - green dots), and left posterior fascicle (LPF - blue dots) were marked prior to ablation of the distal third of the LPF (red/pink dots).

Figures and Legends 


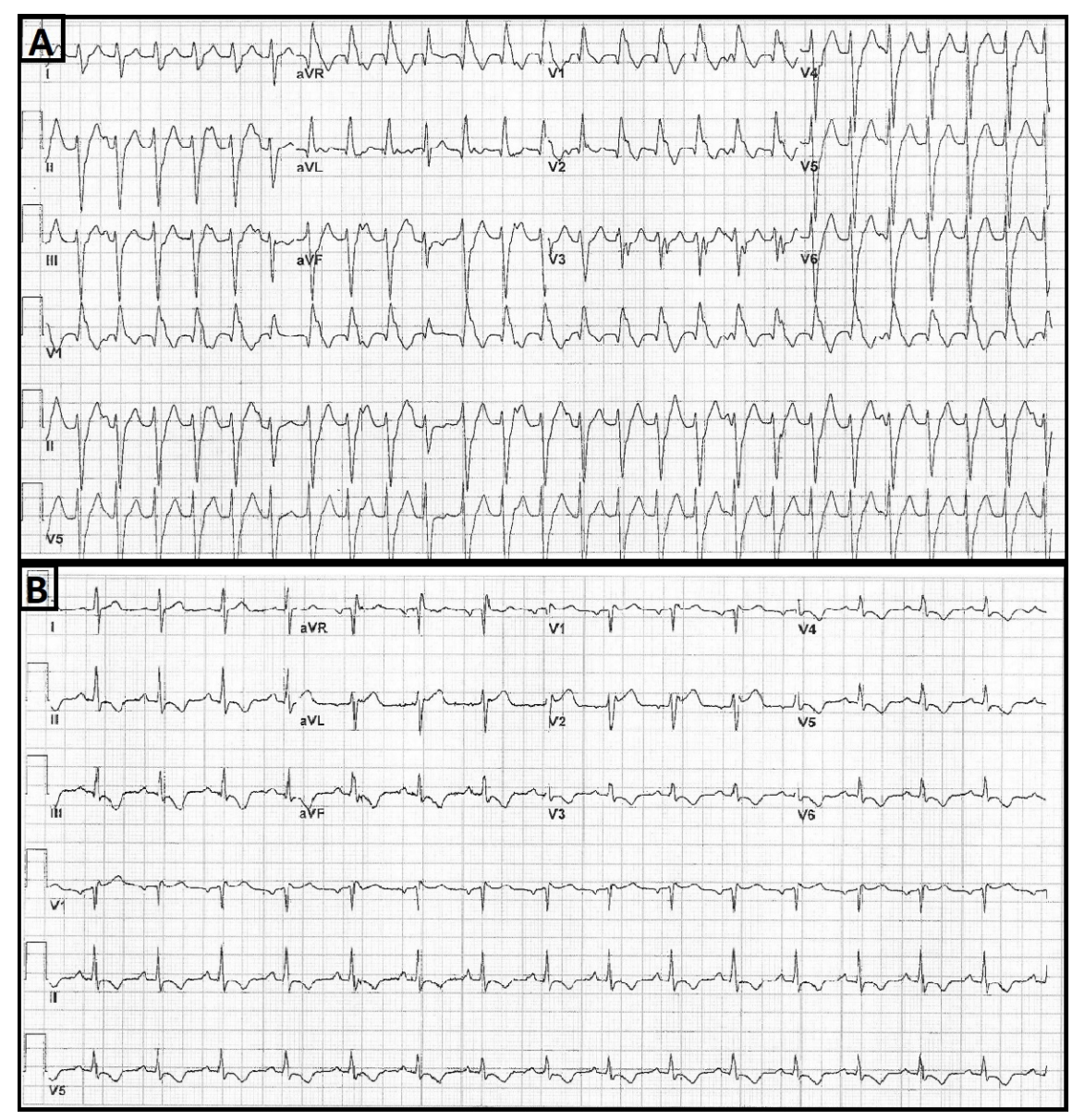

Figure 1. (A) Initial 12-lead ECG obtained at urgent care center. (B) Repeat 12-lead ECG after cessation of tachycardia.

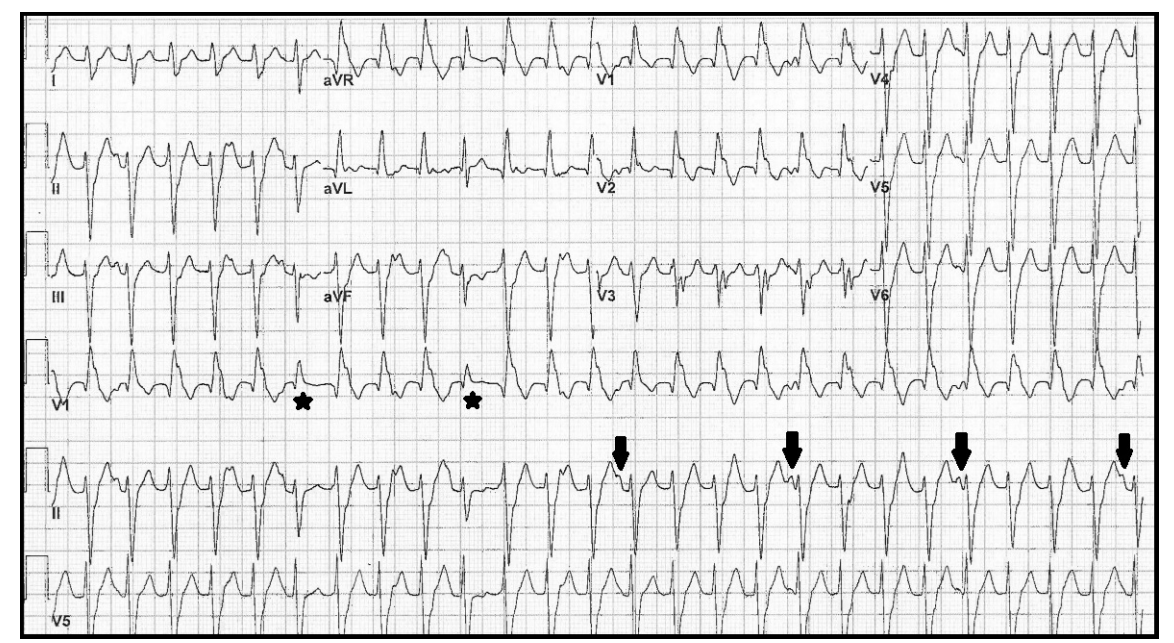

Figure 2. Initial 12-lead ECG demonstrating AV dissociation (arrows) and fusion complexes (stars) making 
ventricular tachycardia (VT) the clear diagnosis.

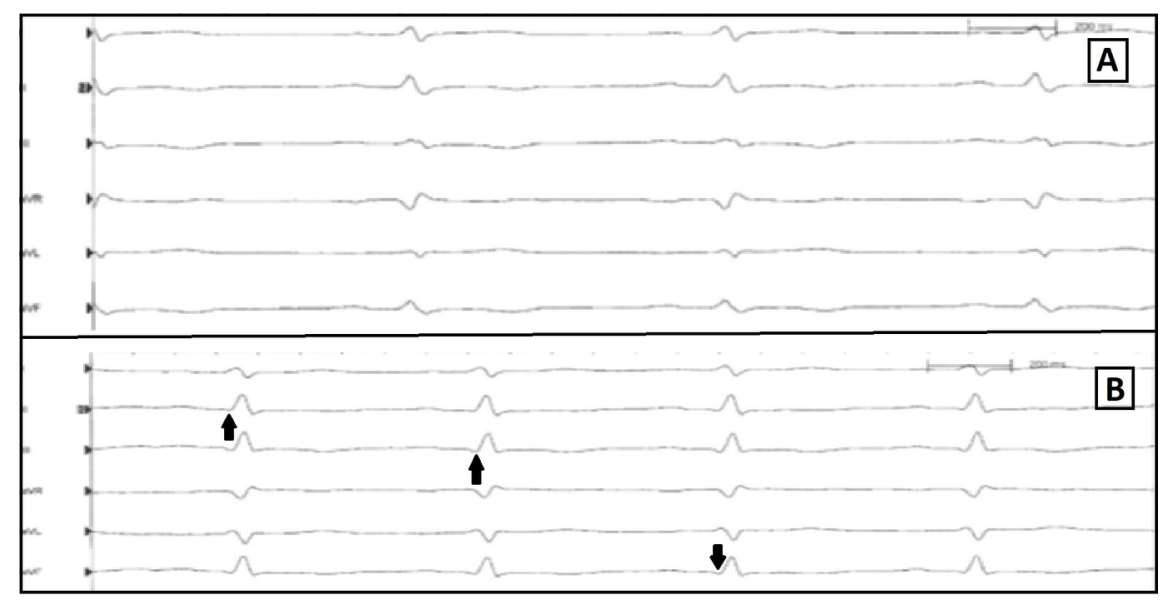

Figure 3. Pre- (A) and post-ablation (B) ECGs demonstrating new q-waves in the inferior limb leads (II, III, and aVF) representing ablation of the distal portion of the left posterior fascicle with initial forces directed superiorly.

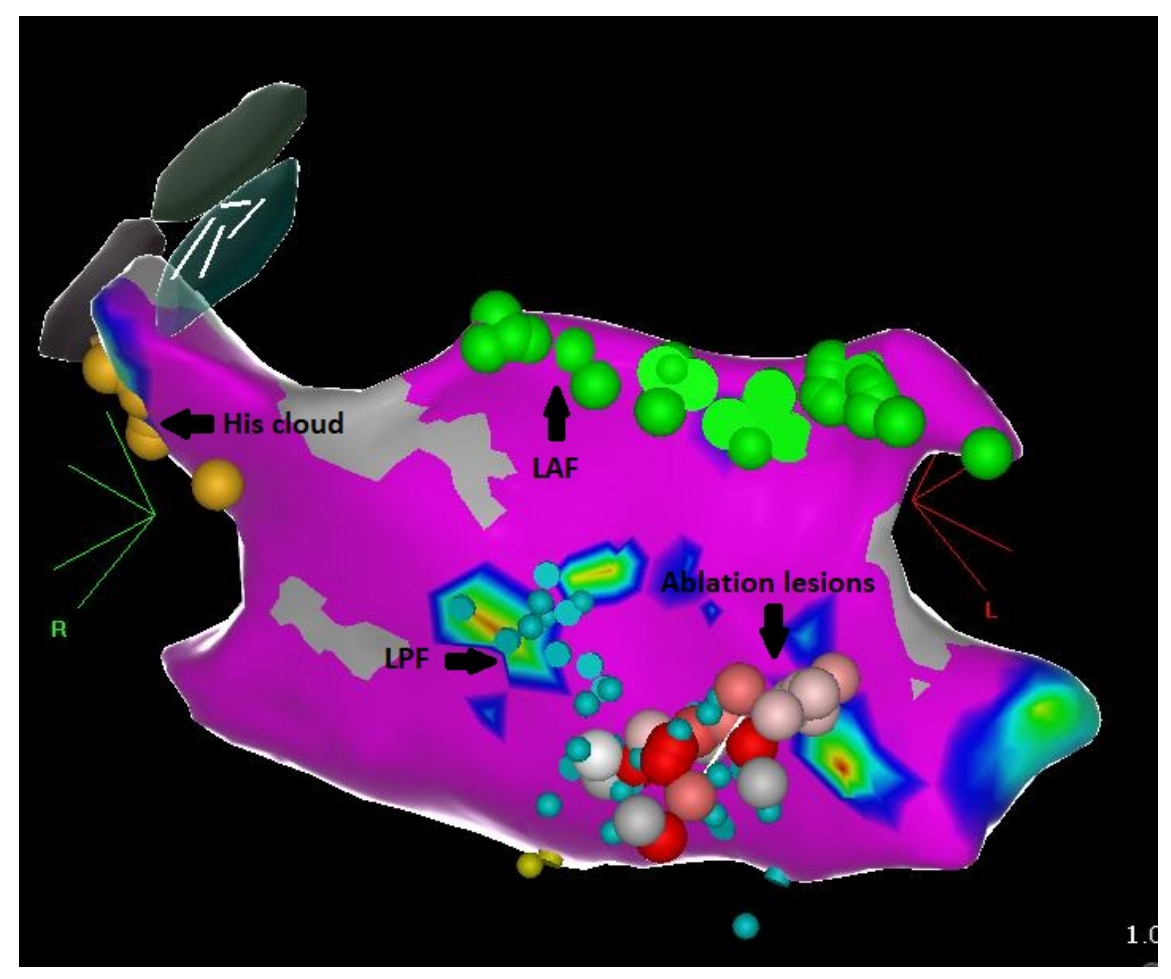

Supplementary Figure. Electroanatomic map of left ventricular septum as viewed from the right anterior oblique projection (RAO). Areas of the His cloud (yellow dots), left anterior fascicle (LAF - green dots), and left posterior fascicle (LPF - blue dots) were marked prior to ablation of the distal third of the LPF (red/pink dots). 


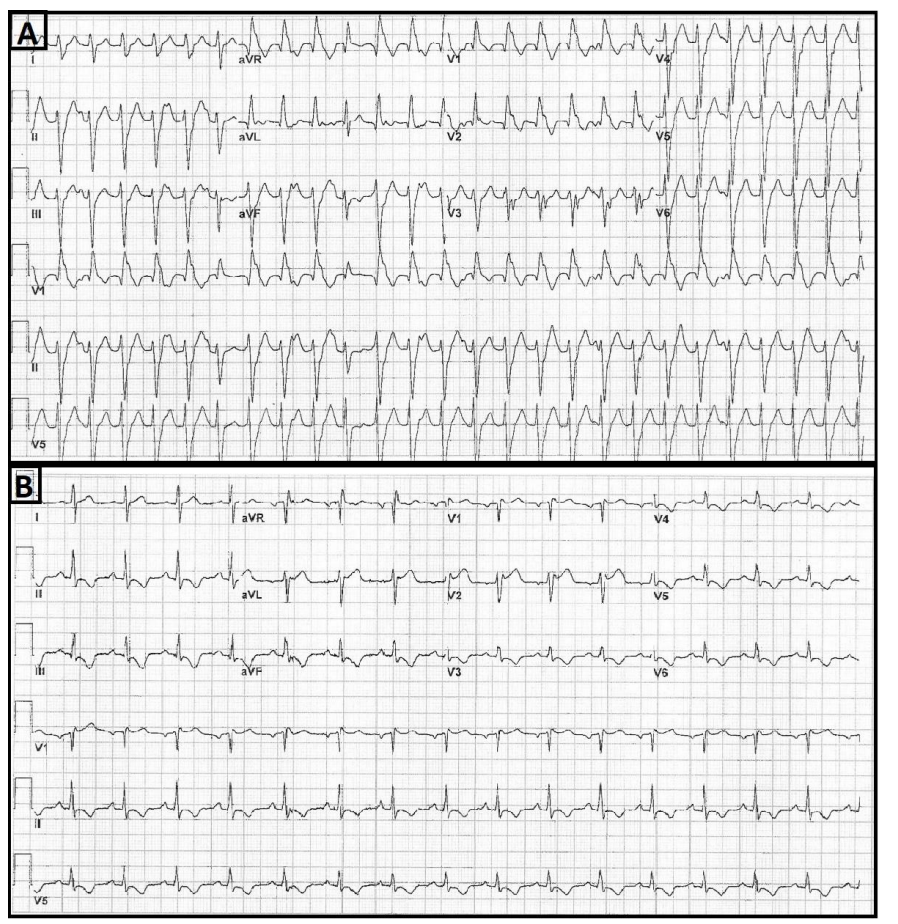

Figure 1. (A) Initial 12-lead ECG obtained at urgent care center. (B) Repeat 12-lead ECG after cessation of tachycardia. 


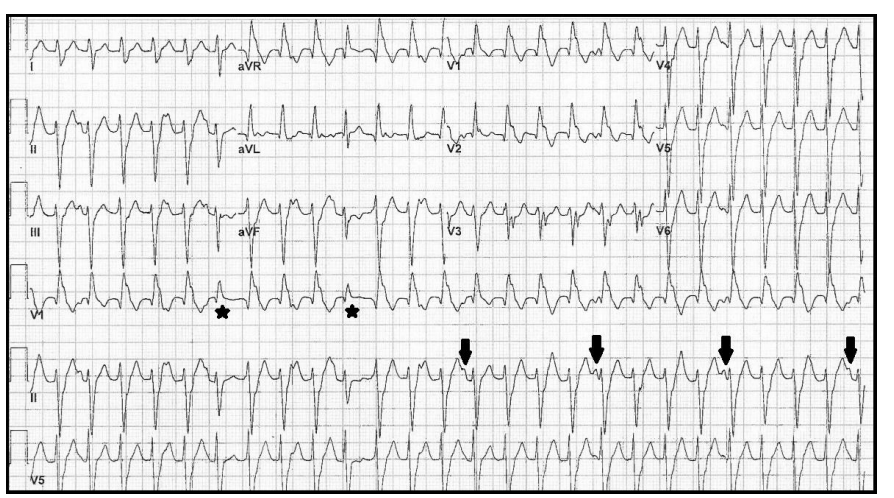

Figure 2. Initial 12-lead ECG demonstrating AV dissociation (arrows) and fusion complexes

(stars) making ventricular tachycardia (VT) the clear diagnosis. 


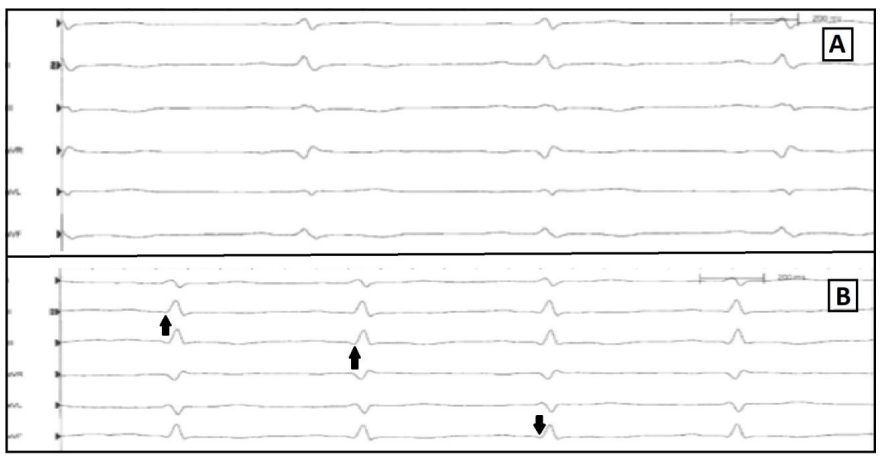

Figure 3. Pre- (A) and post-ablation (B) ECGs demonstrating new q-waves in the inferior limb leads (II, III, and aVF) representing ablation of the distal portion of the left posterior fascicle with initial forces directed superiorly. 